\title{
Magnetic resonance imaging in the CNS
}

\author{
JAMES B. MOORE, MD \\ ABRAHAM PERA, DO \\ NEVA L. FRUMKIN, PhD
}

This article, aimed toward the nonneurologist specialist, presents current indications for MRI in the CNS. Primary and metastatic brain tumors, white matter disease, stroke and hemorrhage, hydrocephalus, and spinal disease are discussed. Emphasis is placed on the contribution of MRI to the evaluation of these major classes of disease. Because of its improved sensitivity and noninvasiveness, in most instances MRI is the initial imaging procedure of choice in the CNS.

Although magnetic resonance imaging (MRI) currently has diagnostic utility for virtually every organ system, the majority of clinical MRI examinations continue to involve the CNS. The procedure is now the preferred initial imaging technique for most patients with CNS complaints. This technology is proliferating at a rapid rate, and all clinicians are finding it desirable to become familiar with the uses and limitations of MRI.

This article, which describes the current applications of MRI in the CNS, is aimed toward the nonneurologic specialist. It is organized according to the major classes of CNS disease, and the figures illustrate the typical MRI appearance of common pathologic entities.

\section{Primary and metastatic brain tumors}

Both primary and metastatic brain neoplasms are visualized easily with MRI, in large part because of the edema that often is associated with these lesions. Magnetic resonance imaging is far more sensitive to vasogenic and cytotoxic edema than computed tomography (CT). ${ }^{1}$ Large tumors are seen well with both modalities, but MRI provides a sub-

stantial gain in detecting small lesions and those located near dense bone. Once a tumor has been demonstrated, the ability to image in multiple planes tremendously aids in precise anatomic localization, which is essential to the neurosurgeon and radiation oncologist as they plan therapy.

A suspected posterior fossa lesion is an important indication for magnetic resonance scanning. Many brainstem neoplasms have densities similar to normal brain and do not exhibit significant contrast enhancement on CT; in addition, high-density streak artifacts often obscure visualization (Fig 1). The MRI characteristics of these tumors demonstrate excellent differentiation from adjacent brain, and no obscuring artifact is caused by the cortical bone (Fig 2). Magnetic resonance imaging readily detects even small intracanalicular lesions (Fig 3 ) because the contents of the internal auditory canal are directly imaged and the petrous bone gives no magnetic resonance signal. Thus, there are no artifacts. Small metastatic lesions in the cerebellar hemispheres are seen equally well.

The middle cranial fossa and high convexity vertex are evaluated better by MRI, again because of high-density streak artifacts on CT. Low-grade high parietal and frontal gliomas, which exhibit vague low attenuation and which demonstrate no contrast enhancement on CT, are diagnosed correctly with MRI. The sella turcica and parasellar regions are particularly well evaluated by MRI, because of the ability of the procedure to obtain direct coronal and sagittal images, improved softtissue contrast, and lack of bone artifacts. ${ }^{2}$

A recent report ${ }^{3}$ has shown even greater sensitivity with MRI when the paramagnetic contrast agent gadolinium-diethylenetriamine pentaaceticacid (Gd-DTPA) is used. ${ }^{3}$ This substance is distributed biologically, much like the iodinated contrast 


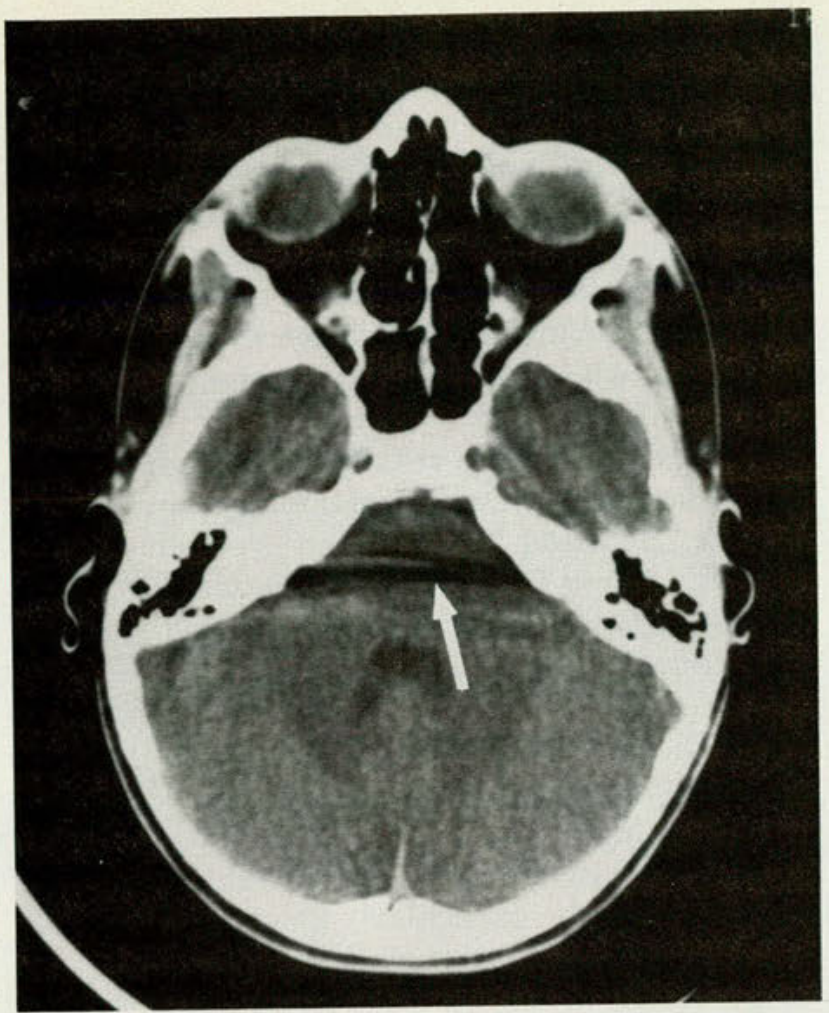

Figure 1. Pontine glioma in 8-year-old girl is obscured by interpetrous, high-density streak artifact (arrow). The only abnormality is minor flattening of anterior fourth ventricle.

Figure 3. Surgically proved intracanalicular acoustic neuroma in 35-year-old man with hearing loss. Although the tumor is of high-signal intensity on this $T_{2}$-weighted MRI image (arrow), many acoustic neuromas have a lower signal.

media used in CT, and it is now available for general use. Suspected metastatic disease to the brain is another common clinical problem that is evaluated best with MRI. The correct assessment of the number of lesions and specific locations has important treatment implications.

\section{White matter disease}

One of the biggest impacts of MRI on neurologic diagnosis has been in the evaluation of white matter disease. The ability to detect demyelinating lesions in multiple sclerosis was recognized early, and MRI now is an important initial test when this diagnosis is suspected. Most of these patients have normal CT findings. The typical magnetic resonance appearance of the disease is multiple focal

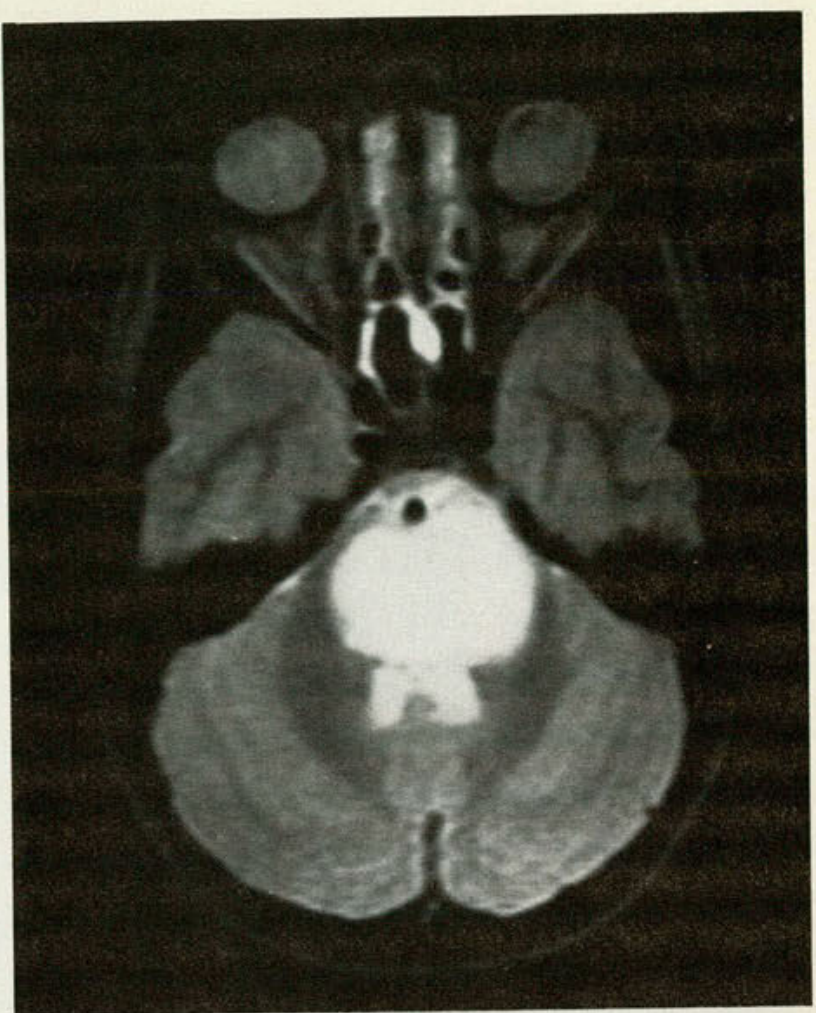

Figure 2. Magnetic resonance scan (TR 2.0 seconds, TE $80 \mathrm{~ms}$ ) obtained at similar level as CT scan in Figure 1 shows tumor involving entire pons and flattening fourth ventricle.

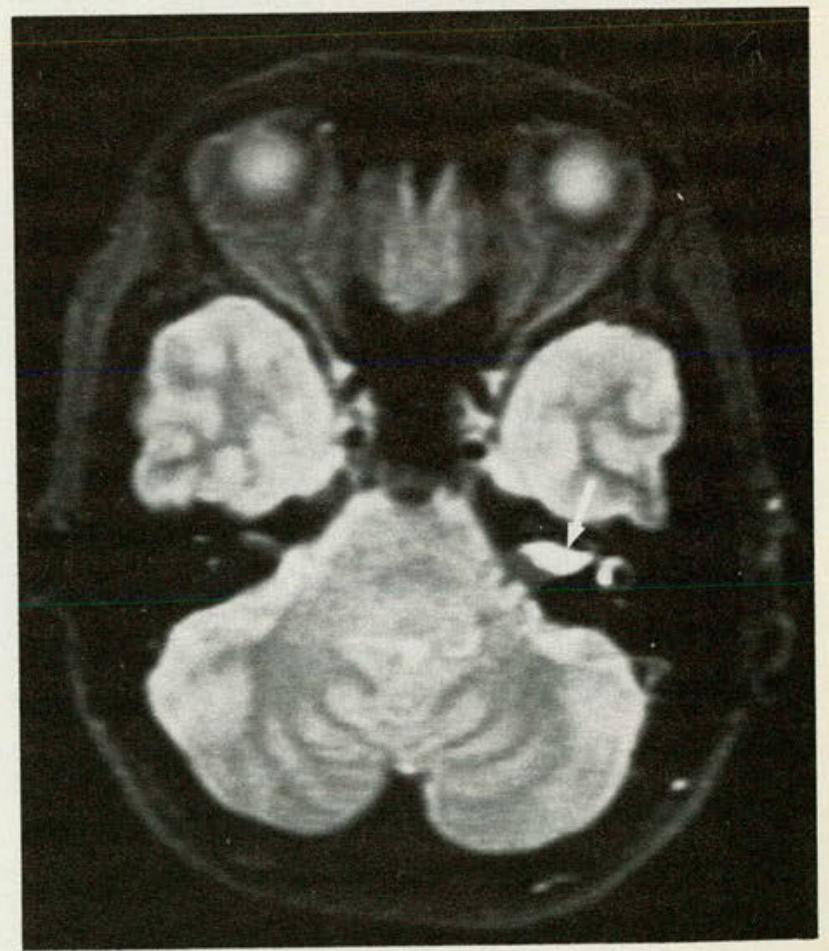




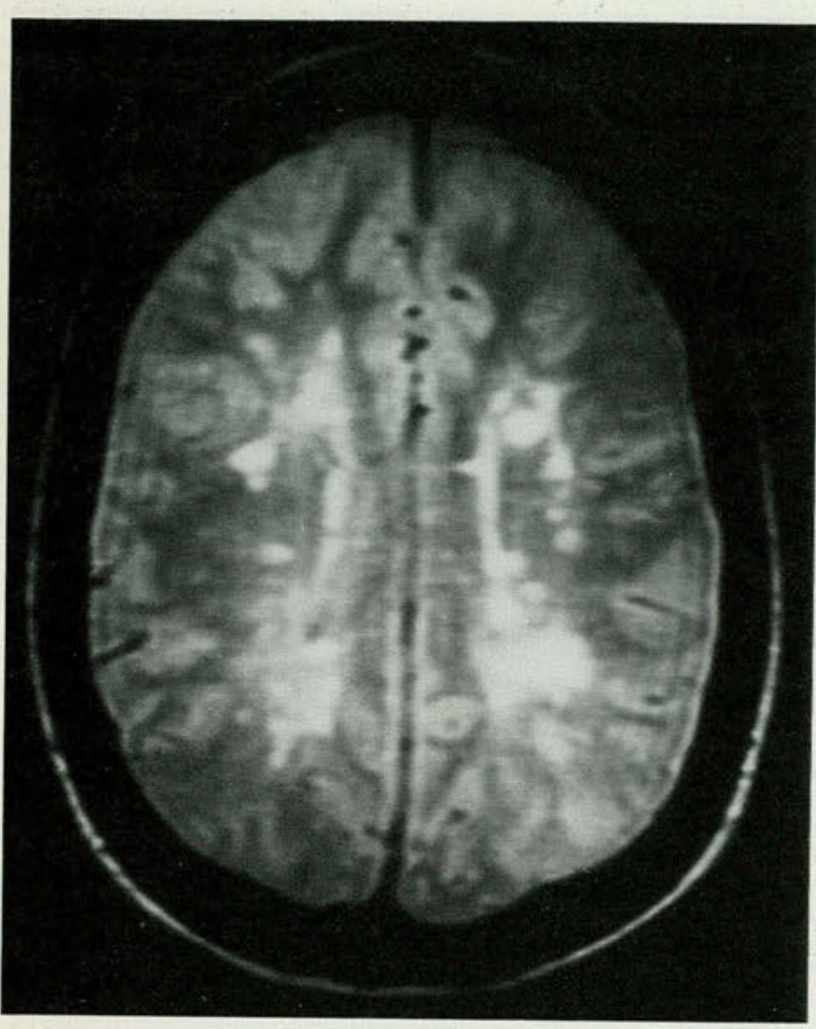

Figure 4. Periventricular and callosal foci with high MRI signal (TR 2.0 seconds, TE $40 \mathrm{~ms}$ ) in this 41 -year-old woman are typical of multiple sclerosis.

areas (with a periventricular predilection) of increased signal intensity on $\mathrm{T}_{2}$-weighted images (Fig 4). Lesions may occur anywhere within the CNS, and specific areas of demyelination often can be correlated with clinical findings (for example, a medial longitudinal fasciculus lesion in the dorsal pons resulting in internuclear ophthalmoplegia). ${ }^{4}$

Secondary demyelinating disease, such as postirradiation and chemotherapy sequelae, can be evaluated equally. Secondary lesions generally are larger and more confluent than those seen in primary demyelinating disease. Magnetic resonance imaging detects early white matter changes that may require therapy adjustments, and it is an excellent tool for following the progress of these patients.

Dysmyelinating conditions, which involve the production of abnormal myelin, are considerably less common than diseases that affect normal myelin. Many of these diseases occur in infants, but milder forms may affect older children or even adults. These disorders are detected easily with MRI.

\section{Stroke and hemorrhage}

Magnetic resonance imaging adds a new dimen- sion to the evaluation of suspected cerebrovascular disease. Its increased sensitivity for vascular events, together with its ability to characterize and stage cerebral hemorrhage, makes MRI the preferred imaging method. However, in the acute setting, CT still may be most appropriate to guide surgical and medical management of the patient. Also, a patient in an unstable condition who requires life support or who is unable to cooperate is examined more easily by CT.

Cortical infarcts are visualized well in both acute and chronic stages because of their increased water content. By using MRI, it often is possible to distinguish tumor from infarct in patients in whom CT reveals only a vague, nonenhancing region of low attenuation (Fig 5). Multiplanar capability and improved anatomic detail help in establishing a vascular etiology (Fig 6).

Small-vessel ischemic events are delineated clearly by MRI; they appear as focal areas of highsignal intensity (Fig 7). Objective evidence of prior infarction can be particularly helpful when evaluating dementia or ataxia of clinically uncertain etiology. Small brainstem infarcts are seen equally well.

Localized brain hemorrhage is especially amenable to MRI examination because of the unique paramagnetic effects of hemoglobin and its degradation products. ${ }^{5}$ These substances undergo a welldefined evolution within the hematoma, and their impact on proton relaxation times allows confident identification and aging of the bleeding.

The sensitivity of MRI to hemorrhage also is useful in evaluating sequelae of head trauma. As in acute stroke, CT is preferred for the severely injured patient in unstable condition, because of faster examination times, ease of providing life support, and sensitivity to cranial and facial fractures. Once the patient's condition is stable, however, MRI is the procedure of choice for staging parenchymal brain and extra-axial blood collections and for detecting small subdural collections and coup-contrecoup phenomena (Fig 8). Magnetic resonance imaging is particularly helpful for following the evolution of cerebral contusion or hematoma and for detecting delayed complications (for example, subdural hematoma) in the deteriorating patient.

Arteriovenous malformations are well delineated because of pathologic features that produce characteristic magnetic resonance patterns. Rapidly flowing blood gives no signal on most pulse sequences; thus, it appears black on MRI. Figures 9 and 10 illustrate these features in the abnormal vessels of a large arteriovenous malformation. 


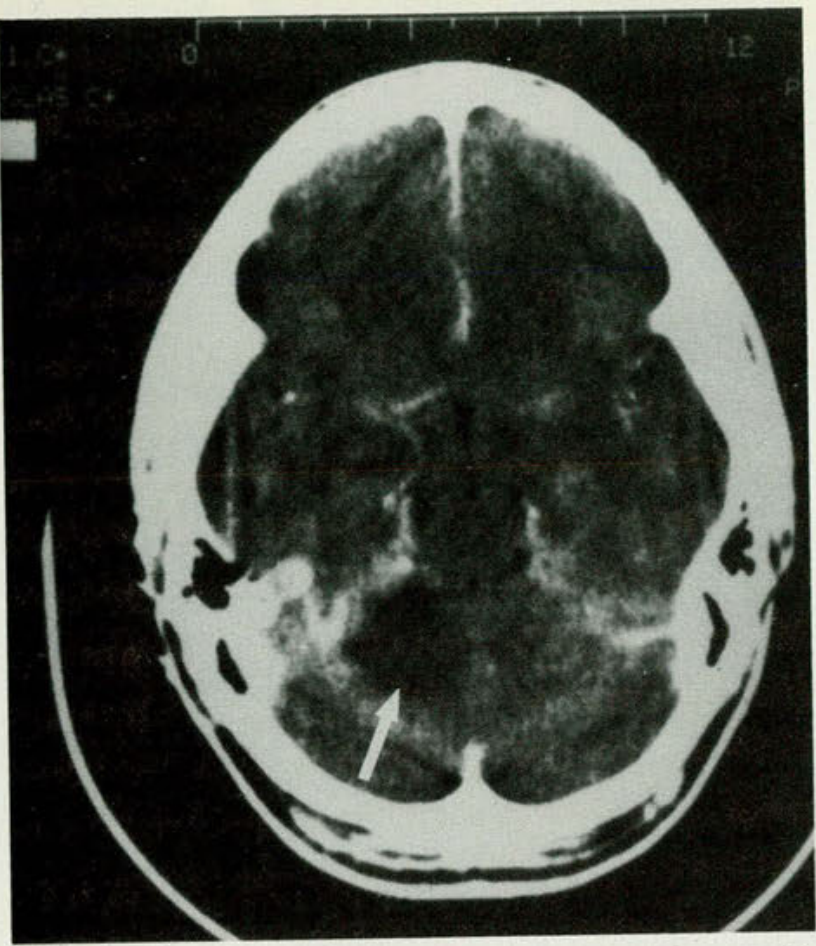

Figure 5. Superior cerebellar infarct in 25-year-old man with acute ataxia. CT scan shows nonenhancing, low-attenuation lesion (arrow), which would be consistent with infarct or tumor.

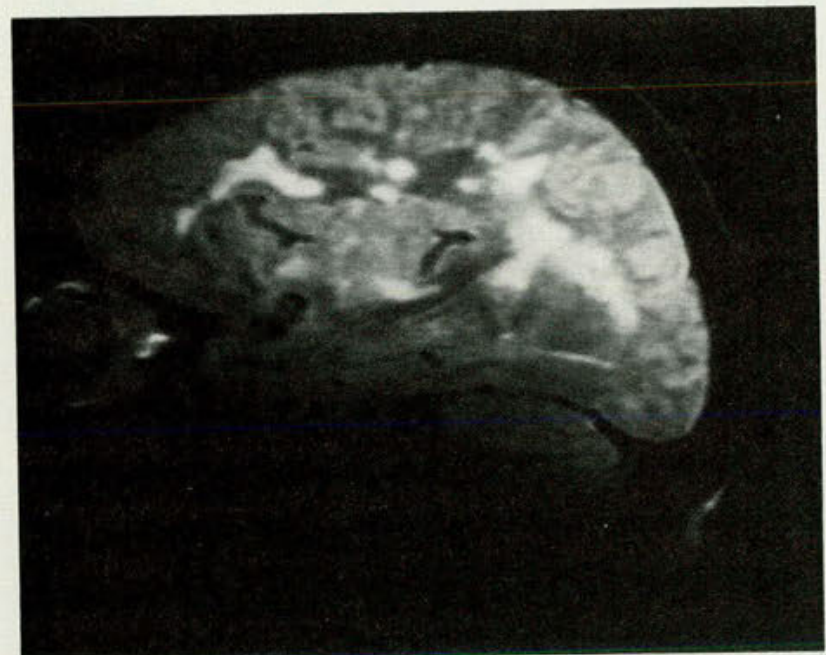

Figure 7. Sagittal section MRI (TR 2.0 seconds, TE $40 \mathrm{~ms}$ ) of 62 -year-old male hypertensive with step-wise progression of dementia demonstrates focal subcortical ischemic events.

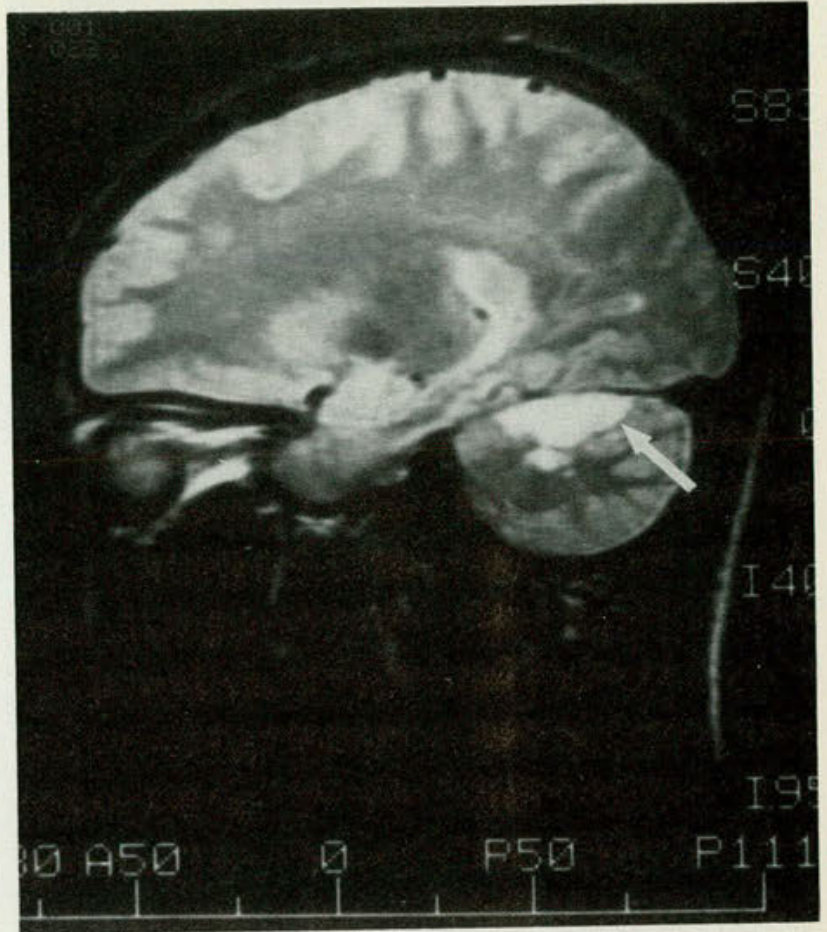

Figure 6. Magnetic resonance image (sagittal section) reveals well-defined, wedge-shaped lesion (arrow) that is confined to vascular distribution. Presumed etiology is migraine.

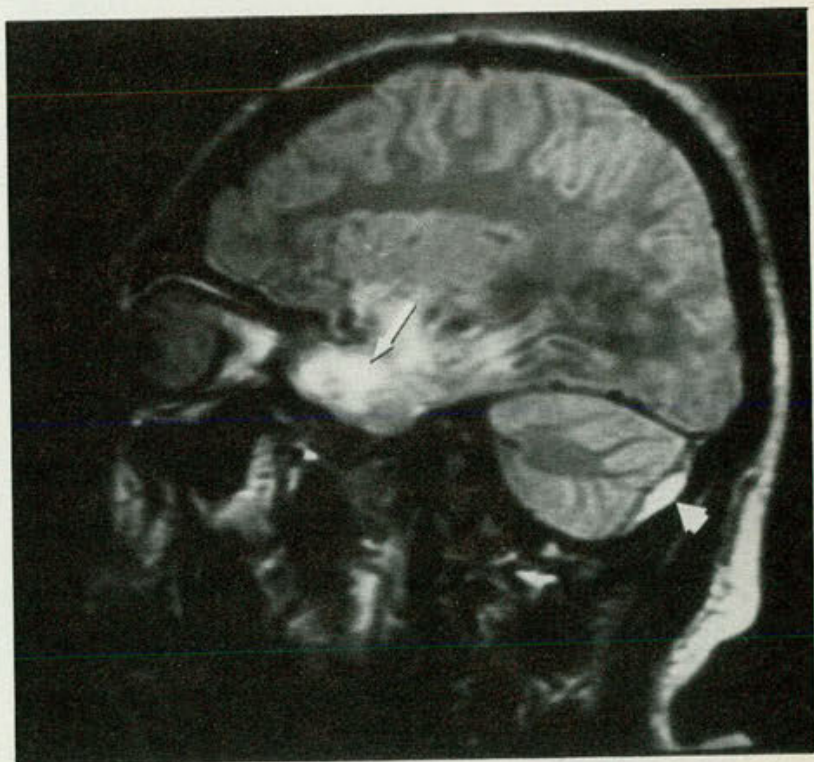

Figure 8. Posttraumatic cerebral contusion and subdural hematoma on MRI. This 18-year-old male patient was struck on the back of the head and suffered temporal lobe contrecoup contusion (arrow) and small posterior fossa subdural hematoma (arrowhead). The subdural hematoma was not seen on CT because of bone artifact. 


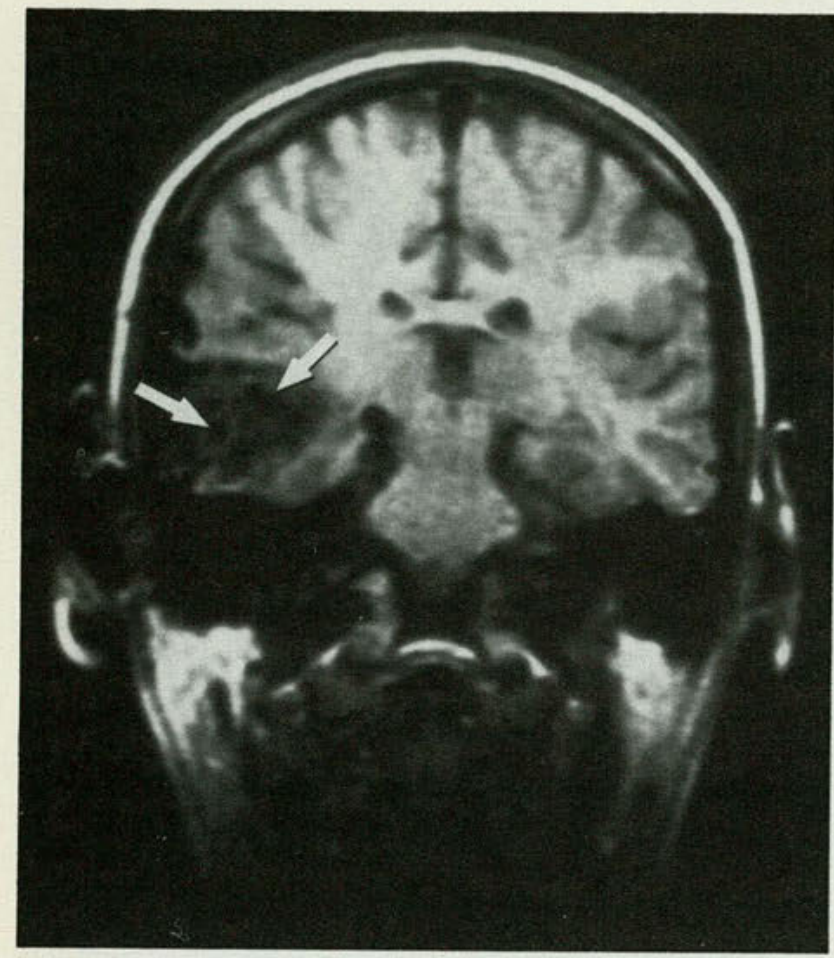

Figure 9. A 25-year-old woman had large, right-sided frontotemporal arteriovenous malformation. Spin echo MRI (coronal section; TR 0.6 seconds, TE $25 \mathrm{~ms}$ ) exhibits absent signal in vessels with rapid flow (arrows).

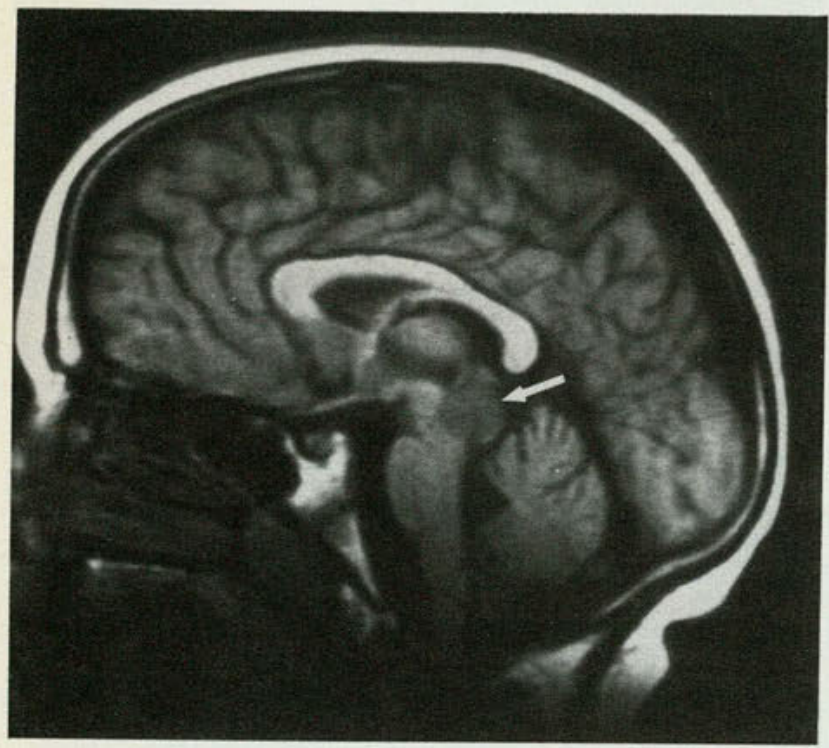

Figure 11. A 12-year-old girl underwent a shunting procedure for hydrocephalus that was presumed to be caused by aqueductal stenosis. Sagittal section MR (TR 0.6 seconds, TE $25 \mathrm{~ms}$ ) shows a well-circumscribed mass arising from tectum of midbrain (arrow), which obstructs the aqueduct. CT did not demonstrate the lesion.

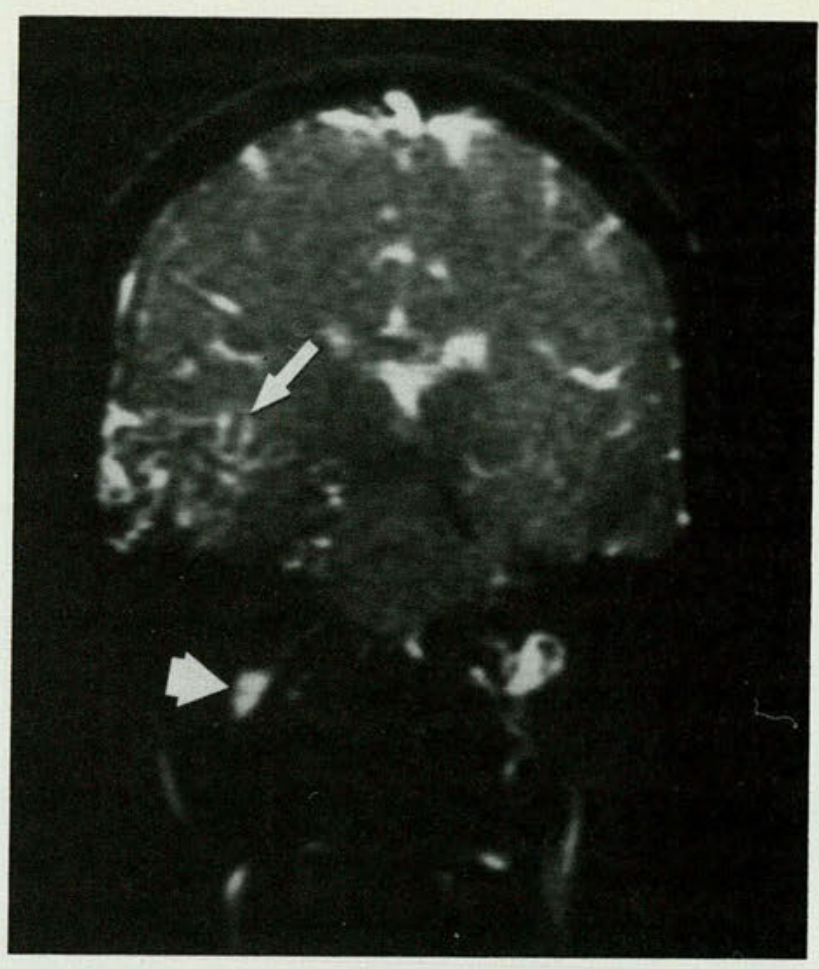

Figure 10. Short flip angle MRI (coronal section) of arteriovenous malformation seen in Figure 9. Note the signal intensity of vessels in the malformation (arrow) and carotid artery (arrowhead) with this pulse sequence.

\section{Hydrocephalus}

Hydrocephalus, or dilatation of the ventricles out of proportion to the size of the sulci, may be caused by a number of pathologic entities. The purpose of imaging patients with hydrocephalus is to establish the diagnosis and determine the underlying cause, as well as to monitor response to shunting and evaluate possible shunt complications.

Computed tomography and MRI are comparable in detecting ventricular dilatation, but MRI clearly is superior in characterizing the underlying pathologic disease and CSF flow dynamics. Masses that cause obstruction in the ventricular system are more sensitively detected in any location; small tumors in and around the aqueduct are evaluated particularly well with MRI (Fig 11). Conversely, the absence of an anatomic or signal abnormality in hydrocephalus allows the confident exclusion of neoplasm as the underlying cause.

In addition to the improved delineation of mass lesions, MRI provides functional information about flow dynamics and pressure within the ventricular system. In acute obstruction, intraluminal ven- 
tricular pressure rises and causes stasis in the subependymal regions around the third and lateral ventricles. This fluid stasis results in a thick rim of high-signal edema surrounding the ventricles (Fig 12) and provides an important clue to ventricular pressure. Conversely, patients with socalled normal pressure hydrocephalus exhibit no surrounding rim of edema, but they often manifest an exaggerated flow void phenomenon in the aqueduct because of decreased ventricular compliance and jetting of CSF through the aqueduct. ${ }^{6}$

Magnetic resonance imaging is superior to $\mathrm{CT}$ for assessing response to shunt therapy in hydrocephalus, as well as for evaluating shunt complications. In the case illustrated by Figures 13 and 14 , for example, the patient experienced a leftsided superior oblique palsy after placement of a shunt at the fourth ventricle. Multiplaned MRI showed the tip of the shunt tube impressing the dorsal pons at the expected location of the proximal fourth cranial nerve. This anatomic relation was not evident on the axial CT scan, and slight withdrawal of the shunt resulted in prompt resolution of the extraocular muscle weakness. Other complications, such as extra-axial fluid collections, are evaluated best by MRI.

\section{Spinal disease}

In the spinal cord, MRI is the initial imaging procedure of choice because of its high sensitivity and noninvasiveness, and most patients will require no further imaging. Earlier strategies for evaluating cord tumors and cavities mainly used myelography and CT, both of which have significant limitations. For example, the magnetic resonance image in Figure 15 clearly defines the superior extent of an ependymoma originating in the cauda equina by sharply contrasting signals. The morphology of

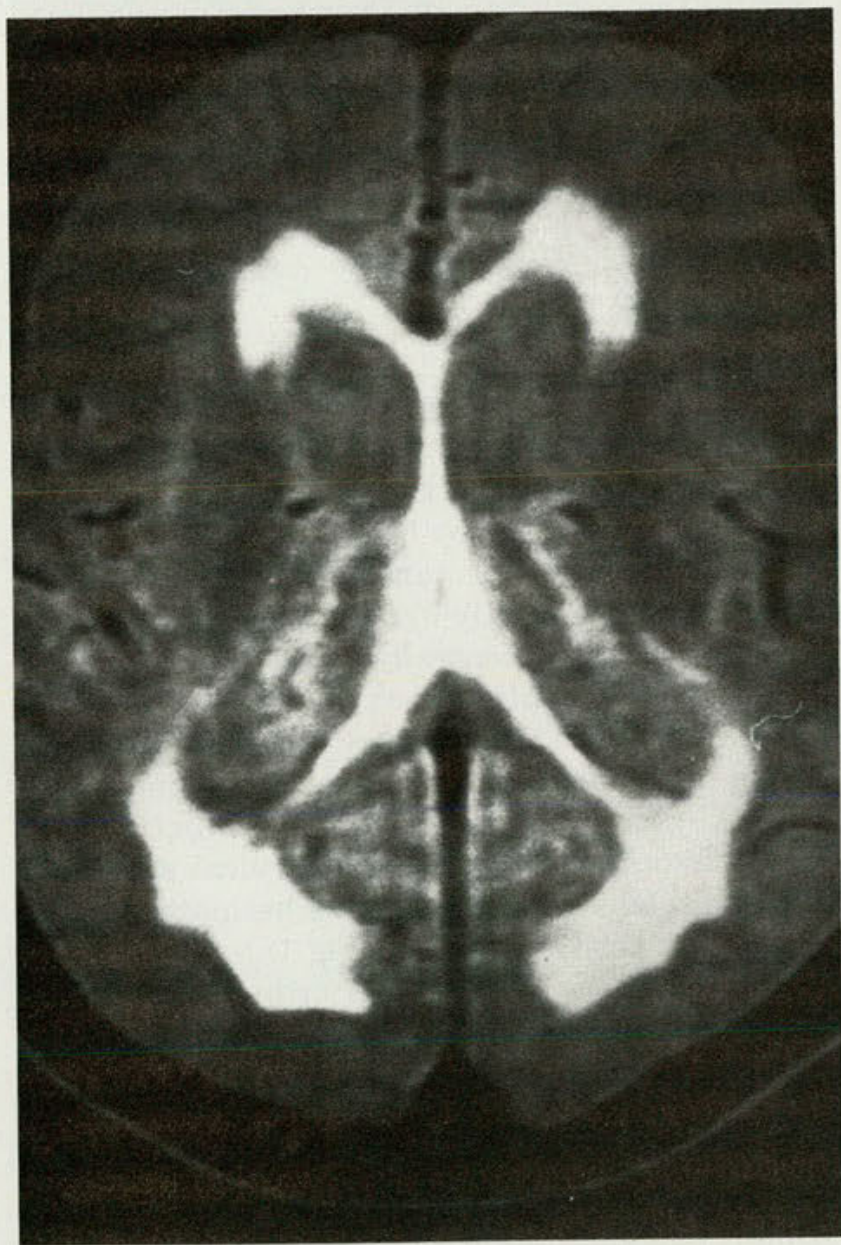

Figure 12. In this 75-year-old man, acute hydrocephalus was caused by obstruction of the fourth ventricle, which was secondary to metastatic lung carcinoma. Left, high-signal edema surrounds

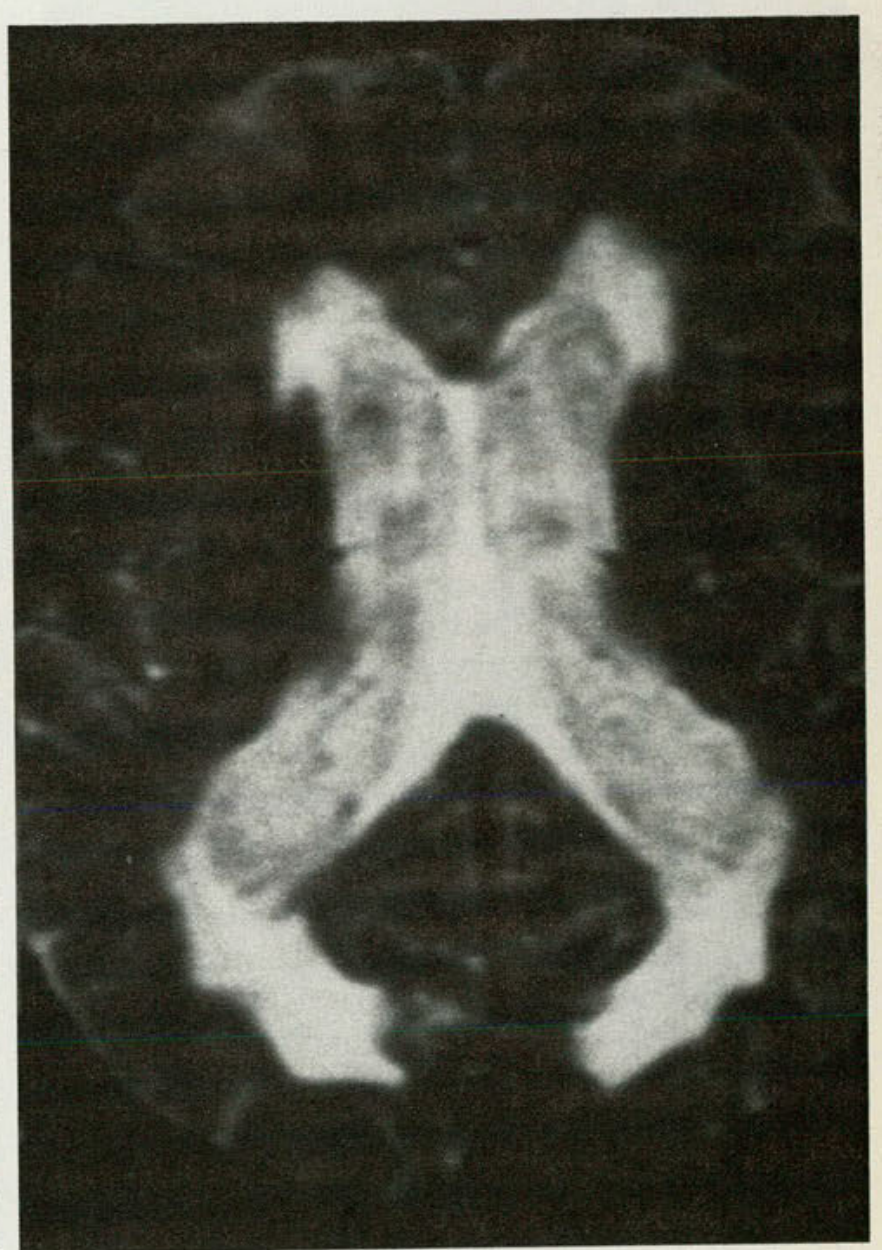

lateral ventricles, which is typical of a high-pressure system. Right, a more $\mathrm{T}_{2}$-weighted image (TR 2.0 seconds, TE $80 \mathrm{~ms}$ ) reveals high-signal intensity CSF. 


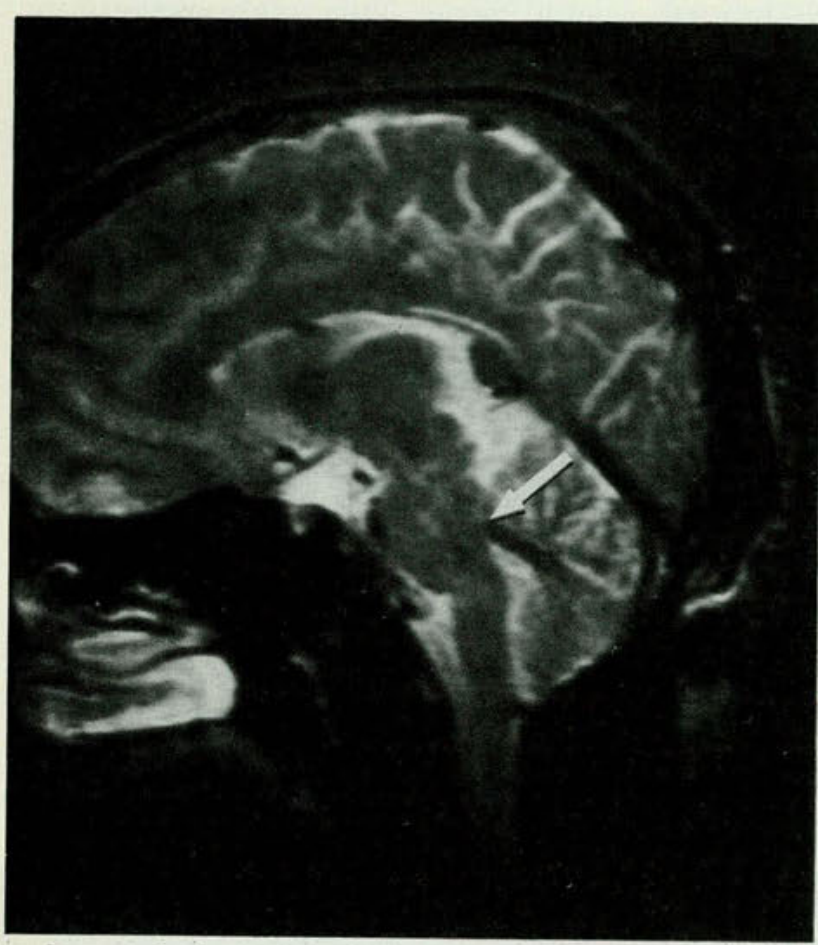

Figure 13. Ventriculoperitoneal shunt, which was inserted for trapped fourth ventricle in this 32-year-old man. The shunt tip (arrow) impinges on the dorsal pons, causing deficit in the fourth cranial nerve.

the tumor is clarified further by an axial section (Fig 16), which shows the abnormal tissue as a thick rind of increased signal intensity enveloping the normal cord. Because of the similar densities of the normal and abnormal tissues, the CT scan (axial section) was unable to detect this morphologic pattern of tumor surrounding the cord. Smaller, more focal intramedullary lesions that exert no mass effect also are seen easily, thus illustrating the utility of MRI in the evaluation of any myelopathy.

The relative utility of MRI for imaging extradural lesions (disk herniation, hypertrophic bone, metastases) varies mainly with location and level within the spine. In the lumbar spine, MRI has closer correlation with surgical findings than either myelography or CT alone, ${ }^{7}$ although these modalities often are complementary. Facet joint hypertrophy is seen better with CT.

Radiculopathy is evaluated well by MRI because the nerve root canals and their contents are delineated clearly on the more lateral sagittal images (Fig 17). Stenosis of a foramen is detected easily, but it may not be possible to determine the

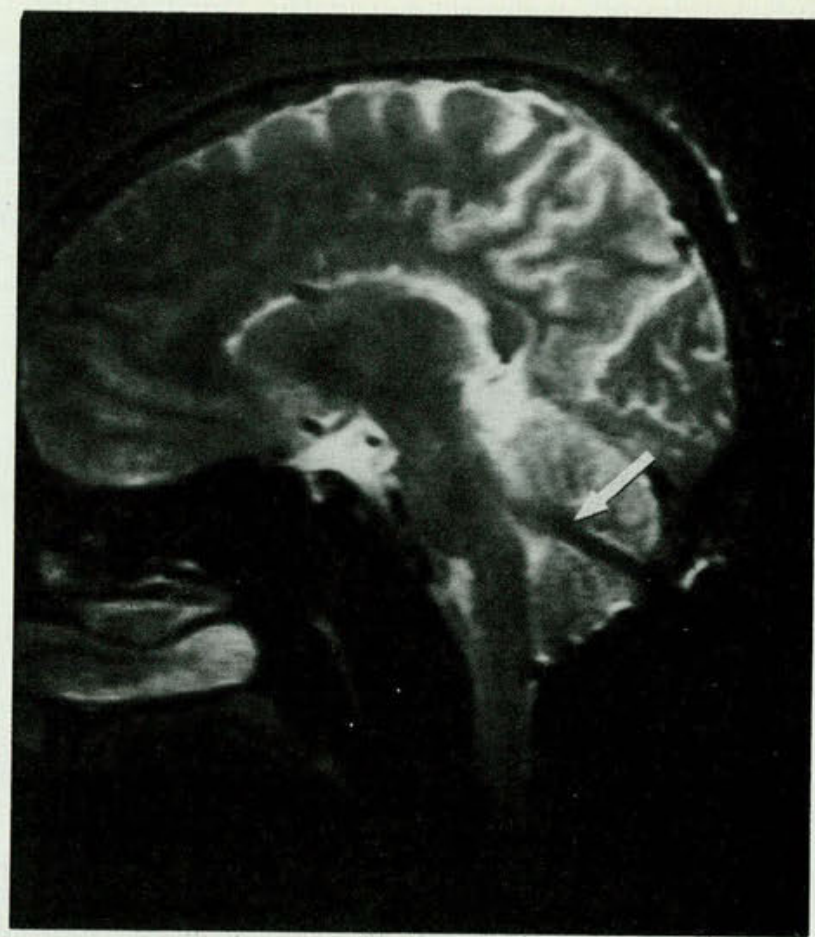

Figure 14. With the shunt (Fig 13) pulled back, the patient's palsy resolved.

precise nature of the lesion (for example, degenerated disk or bony spur). In general, however, lateral extradural disease that results in radiculopathy is evaluated best by MRI of the lumbar spine. Encroachment of the foramen in the cervical spine is not imaged as easily by MRI, because the nerve root canals angle ventrally at 45 degrees, which makes it impossible to look "down the barrel" of the canal with a parasagittal image.

High-resolution CT with intrathecal contrast remains an important modality for evaluating subtle extradural disease in the cervical spine, although the usual cervical spine herniated disk is visualized easily with MRI (Fig 18). At all spinal levels, however, MRI is superior in detecting and determining the extent of bony and soft-tissue metastatic disease.

As in the brain, congenital abnormalities of the spine often are delineated better by MRI because of its ability to image in multiple planes. The Chiari I malformation, for example, traditionally has required an invasive, time-consuming evaluation, which now is obviated completely by MRI. The relationship of the cerebellar tonsils to the in- 


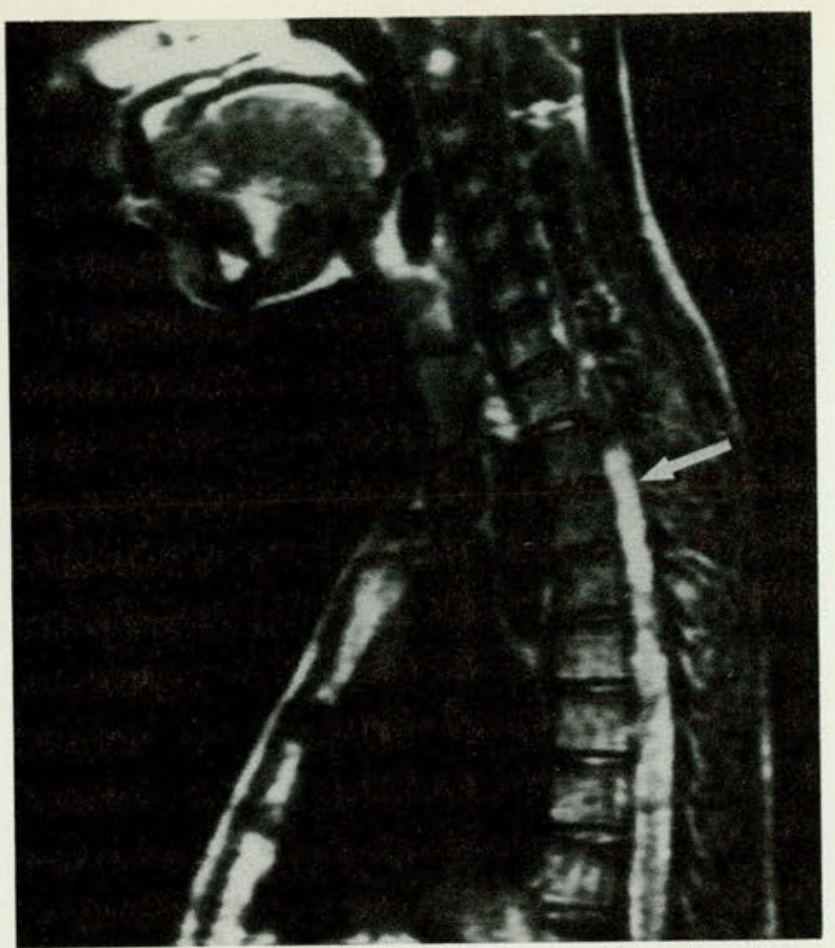

Figure 15. Filum terminale ependymoma in a 15-year-old boy. In this MRI (TR 1.5 seconds, TE $35 \mathrm{~ms}$ ), the tumor is seen to have grown upward to the D-2 level (arrow).

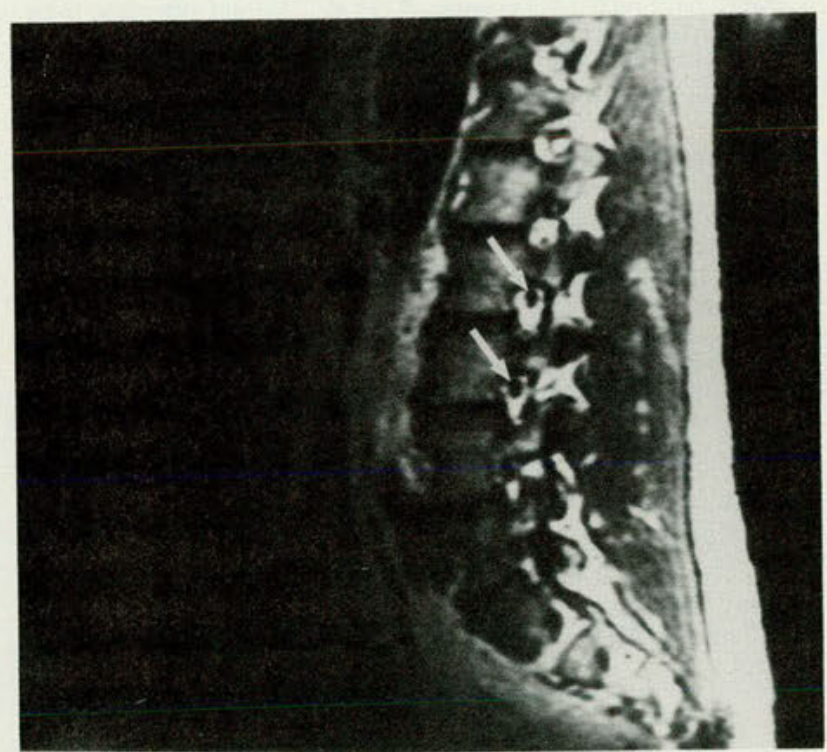

Figure 17. Lumbar spine MRI (parasagittal section) shows nerve root canals to best advantage. Fat is of high-signal intensity on this $T_{1}$-weighted acquisition (TR 0.6 second, TE $25 \mathrm{~ms}$ ) and outlines exiting nerve roots (arrows).

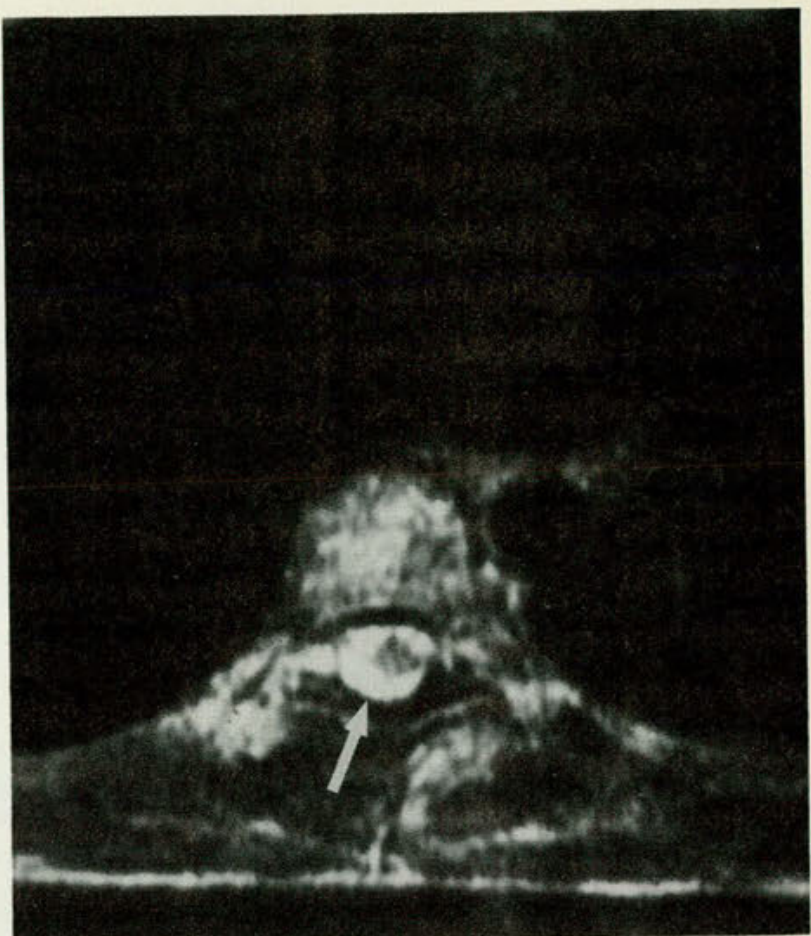

Figure 16. Axial section MRI (TR 1.5 seconds, TE $35 \mathrm{~ms}$ ), shows high-signal tumor (arrow) enveloping the spinal cord; this is a common morphologic pattern with this lesion.

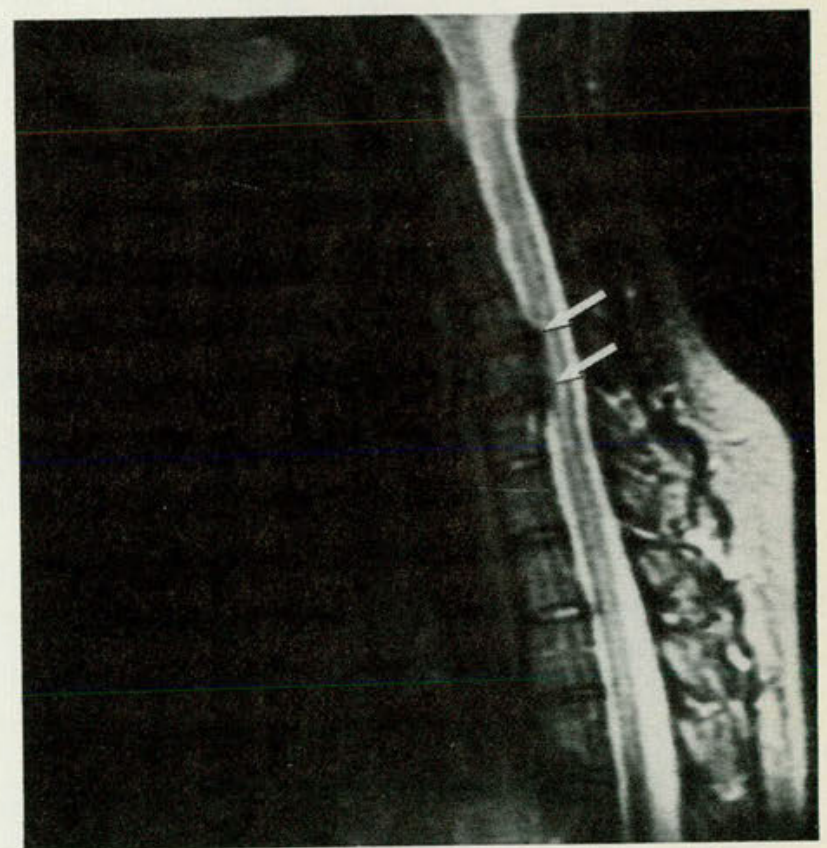

Figure 18. Central herniations of $C-5$ to $C-6$ and $C-6$ to $C-7$ cause obvious extradural impressions on the thecal sac (arrows). The CSF is of high signal on this $T_{2}$-weighted image (TR 2.0 seconds, TE $80 \mathrm{~ms}$ ), which gives a "myelogram effect"(ie, the CSF white and the cord dark as seen in a myelogram). 
Figure 19. Chiari I malformation. Cerebellar tonsils are displaced below foramen magnum (arrow), and there is a large associated hydromyelia (arrowhead). The CSF is low signal on this $T_{1}$-weighted image.

ferior aspect of the foramen magnum is determined easily, as is the presence of any associated syrinx (Fig 19). Most other craniovertebral junction lesions, including tumor, are evaluated best with MRI.

\section{Conclusion}

Magnetic resonance imaging currently is making a tremendous contribution to the diagnosis of neurologic disorders, and the future development of new, specialized pulse sequences and contrast agents promises even more exciting applications. The increased sensitivity of MRI as compared with CT makes it the initial imaging procedure of choice for the majority of patients with CNS signs or symptoms, particularly for patients whose clinical findings are subtle or equivocal. CT remains helpful in characterizing certain lesions once they have been discovered by MRI, but there are few instances when CT better detects CNS disease. A corollary to this improved sensitivity is that the normal MRI scan excludes many diagnostic possibilities (for example, tumor or demyelination) with a high degree of confidence. This valuable imaging modality helps to decrease physician and patient uncertainty in many cases that have been diagnostic and management dilemmas.

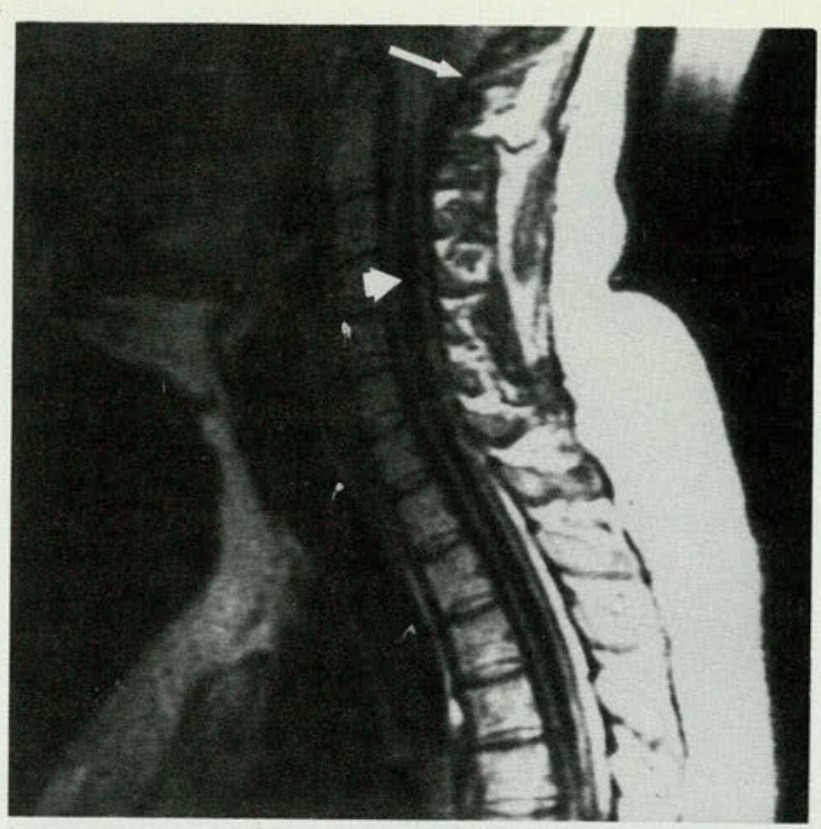

1. Brant-Zawadzki M, Pereira B, Weinstein $P$, et al: $M R$ imaging of acute experimental ischemia in cats. AJNR 1986;7:7-11.

2. Kucharczyk W, Davis DO, Kelly WM, et al: Pituitary adenomas: Highresolution MR imaging at 1.5T. Radiology 1986;161:761-765.

3. Dwyer AJ, Frank JA, Doppman JL, et al: Pituitary adenomas in patients with Cushing disease: Initial experience with Gd-DTPA-enhanced MR imaging. Radiology 1987;163:421-426.

4. Edwards MK, Farlow MR, Stevens JC: Multiple sclerosis: MRI and clinical correlation. AJR 1986;147:571-574.

5. Gomori JM, Grossman RI, Goldberg HI, et al: Intracranial hematomas: Imaging by high-field MR. Radiology 1985;157:87-93.

6. Bradley WG Jr, Kortman KE, Burgoyne B: Flowing cerebrospinal fluid in normal and hydrocephalic states: Appearance on MR images. Radiology 1986;159:611-616.

7. Modic MT, Masaryk T, Boumphrey F, et al: Lumbar herniated disk disease and canal stenosis: Prospective evaluation by surface coil MR, CT, and myelography. AJR 1986;147:757-765.

From the Department of Radiology, Michigan State University College of Osteopathic Medicine, East Lansing, Mich.

Reprint requests to Dr Pera, B220 Clinical Center, MSUCOM, East lansing, MI 48824 